\title{
Studies on the Karyotype of the Black Rat, Rattus rattus, Collected at the University of Nigeria Zoological Garden
}

\author{
Amaka, John Ifeanyichukwu \\ Department of Zoology, University of Nigeria, Nsukka, Nigeria
}

\begin{abstract}
Karyotypic studies were carried out on the Black Rat, Rattus rattus, with the aim of determining its chromosome number. The chromosomes were prepared from the bone marrow of a male rat treated with $0.04 \%$ colchicine. Chromosomes in well spread cells were counted and measured using Motic Images Plus - a computer software. Arm ratios and centromeric indices were determined from these measurements. The chromosomes were classified based on the centromeric indices obtained. Ideograms were also constructed from the measurements. Photomicrographs of well spread mitotic metaphase chromosomes were used to construct a standard karyotype for the species. A diploid chromosome number of $2 n=38$ with a fundamental number (FN) of 76 was obtained for the local population of the Black Rat, Rattus rattus, here in the University of Nigeria, Nsukka. The karyotype shows a somewhat sharp break in chromosome lengths between chromosomes 4 and 5 , and thereafter, a gradual decrease in size. From the constructed idiogram, there is a gradual decrease in length from one chromosome pair to another. The X and Y complements sharply deviated from this trend.
\end{abstract}

Keywords: chromosome, diploid number, karyotype, longarm, shortarm

\section{Introduction}

The term "rat" generally applies to all medium-sized, long-tailed rodents of the super-family, Muroidea. "True rats" are members of the genus Rattus, the most important of which are the Black Rat, Rattus rattus, and the Brown rat, Rattus norvegicus, which are collectively called the Old World Rats [1].

Rats are typically distinguished from mice by their size: rats are generally large muroid rodents, while mice are small muroid rodents. The muroid family is very large and complex, and the common terms rat and mouse are not taxonomically specific. Generally, when someone discovers a large muroid, its common name is the term rat (genus - Rattus), while if it is small, the name is the term mouse (genus - Mus) [1].

\subsection{Rat Karyotype}

In recent times, there has been much interest in cytological studies of different species of organisms, especially vertebrates. This has lead to the completion of genome sequencing in most of these species. Comparative chromosome studies in related species have been of great value for the establishment of systematic relationships in many plants and animals [2].

A constant chromosome number of $2 n=42$ was established for the Brown Rat, Rattus norvegicus, in 1972 [3]. However, various studies on the chromosomes of different populations of the Black Rat, Rattus rattus, in different locations of the world have portrayed a variation in the chromosome number, ranging from $2 \mathrm{n}=38$ to $2 n=42$. Several authors for instance, [4], [5], [6] and [7], obtained the diploid number of $2 n=42$ for the Japanese populations of the Black Rat. Other authors [8] and [9] researched on the karyotype of the Black Rat populations of Switzerland and Malaysia and found a diploid number of $2 n=42$. A chromosome number of $2 n$ $=38$ was obtained for two South American populations of Rattus rattus [10]. Also, a diploid chromosome number of $2 n=38$ was reported for an Italian population of Rattus rattus [11]. Presently, there is paucity of information on the cytology of black rat races in Africa.

Table 1: Chromosome number of Some Black Rat Populations

\begin{tabular}{ccc}
\hline Author(s)/year & Country/location & Chromosome number \\
Bianchi et al. (1969) & South America & $2 \mathrm{n}=38$ \\
Capanna et al. (1970) & Italy & $2 \mathrm{n}=38$ \\
Capanna and Civitelli (1969) & Italy & $2 \mathrm{n}=38$ \\
Makino (1943) & Japan & $2 \mathrm{n}=42$ \\
Matthey (1956) & Switzerland & $2 \mathrm{n}=42$ \\
Oguma (1935) & Japan & $2 \mathrm{n}=42$ \\
Tateishi (1935) & Japan & $2 \mathrm{n}=42$ \\
Yong (1969) & Malaysia & $2 \mathrm{n}=42$ \\
Yoshida et al. (1965) & Japan & $2 \mathrm{n}=42$ \\
\hline
\end{tabular}


Studies on the Karyotype of the Black Rat, Rattus rattus, collected at the University of .....

\subsection{Objective of Study}

A perusal of available literature did not reveal any record of cytological study of the Nigerian population of Rattus rattus. The aim of this research work, therefore, is to determine the chromosome number of the population of the Black Rat, Rattus rattus, found within the University of Nigeria, Nsukka campus, Enugu State, Nigeria. It is hoped that this will provoke more effort in the cytological studies of different populations of the Black Rat, Rattus rattus, in different regions of Nigeria, and indeed Africa, so as to ascertain their actual cytotaxonomic status.

\subsection{Source of Specimen and Extraction of Cells}

\section{Methodology}

The male Black Rat specimen used was collected from a store house at the Zoological Garden, University of Nigeria, Nsukka. The live specimen was taken to the laboratory and injected intraperitoneally with $2 \mathrm{ml}$ of $0.04 \%$ colchicine solution prepared with sterile physiological saline. After a period of 3 hours of injection, the specimen was sacrificed using chloroform.

Dividing cells were obtained from the marrow of the femur and humerus, dissected out in accordance with the methodology described by [13] and [14]. Both ends of the femur and humerus were cut open, and a hypodermic needle attached to a syringe containing $1-1.5 \mathrm{ml}$ of freshly prepared and pre-warmed $\left(37^{\circ} \mathrm{C}\right)$ hypotonic buffer $(0.55 \% \mathrm{Kcl})$ was inserted. The marrow was aspirated into a $15 \mathrm{ml}$ centrifuge tube. Fat lumps were removed with a pasture pipette. The preparation was left to stand in the hypotonic buffer for not more than 10 minutes.

\subsection{Fixation of Cells}

The tubes were balanced using the hypotonic buffer and centrifuged at 1,000 revolutions per minute (r.p.m) for 5 minutes at room temperature. The supernatant was carefully removed using a suction pipette, remaining a small quantity of about $0.3-0.5 \mathrm{ml}$ in which the cells were suspended. The tubes were shaken briskly so that sediment mixes with the remaining supernatant to form a cell suspension.

Freshly prepared fixative (1: 3 glacial acetic acid - methanol) was added drop wise, with quick agitation after each drop to re-suspend the cells. A total of $2.0-2.5 \mathrm{ml}$ of the fixative were added. The suspension was centrifuged at 1,000 r.p.m for 5 minutes and the supernatant removed. The above process was repeated for two more times and the cells were re -suspended in the fixative.

\subsection{Spreading of Cells}

After the last centrifuging, the cells were re-suspended in appropriate volume of the fixative to give an adequate cell suspension for spreading on the slides. The cells were spread on clean slides by holding the slides at an angle of about $45^{\circ}$. And from a height of about 1 meter, two or more drops of the cell suspension were allowed to fall on the slides uniformly. The slides were blown quickly across its length and placed on a slide warmer set at $60^{\circ}$, and were allowed to dry for about 24 hours before staining.

\subsection{Preparation of Giemsa Stain}

$0.5 \mathrm{~g}$ of Giemsa powder was dissolved in $33 \mathrm{ml}$ glycerol and put in Erlmyer bottle in a dark compartment over night. The next day, it was heated in a water bath set at $60^{\circ} \mathrm{C}$ for 2 hours and was allowed to cool, after which $33 \mathrm{ml}$ of methanol was added. This solution was stored in an amber coloured bottle as the stock Giemsa stain.

\subsection{Staining of Cells}

$5 \mathrm{ml}$ of stock Giemsa stain was diluted with $50 \mathrm{ml}$ of phosphate buffer. The buffer was prepared fresh by simultaneously pouring $25 \mathrm{ml}$ each of Potassium dihydrogen orthophosphate $\left(\mathrm{KH}_{2} \mathrm{PO}_{4}\right)$ and Disodium hydrogen orthophosphate $\left(\mathrm{Na}_{2} \mathrm{HPO}_{4}\right)$ into a coupling jar/chamber. $5 \mathrm{ml}$ of the stock Giemsa stain was now added to it. The resulting stain was stirred with a glass rod to obtain a uniform mixture after which the stain was reduced to an adequate level so as to avoid covering the labels on the slides. The slides were stained for 20 minutes, and rinsed in distilled water to remove the stain. The slides were dropped back on the slide warmer to dry any adhering water molecule.

\subsection{Chromosome Analysis}

The slides were scanned for dividing cells systematically from one end to another under the $\times 10$ objective of light microscope. The objective was changed to $\times 40$ once a likely metaphase cell was spotted. Well spread cells were studied intently under $\times 100$ (oil immersion).

Photomicrographs of the chromosomes were taken under oil immersion. The chromosome's entire length \{long arm (p) as well as the short arm (q) $\}$ was measured with the aid of Motic Images Plus - a computer 
software. The centromeric index (i) was calculated. The classification of the chromosomes based on centromere position follows that proposed by [15]. Appropriate ideogram (karyotype) representing the entire chromosome length and morphology was constructed.

\section{The Karyotype of Rattus rattus}

\section{Results}

After the examination of over 50 well spread mitotic metaphase cells from the bone marrow, a diploid chromosome number of $2 \mathrm{n}=38$ with a fundamental number (FN) of 76 was established for the male Rattus rattus. Figure 1 shows the mitotic metaphase chromosome spreads for the male Rattus rattus, while figure 2 portrays the karyotype.

From five well spread metaphase cells, the total lengths of the chromosomes as well as the lengths of the arms were measured under oil immersion using Motic Images Plus. Table 2 shows the chromosome measurements, centromeric indices and their nomenclature. From the measurements of the chromosomes, the karyotype may be divided into three distinct groups according to size. Group 1 consists of the large chromosomes numbered 1 to 4 . Structurally, this group comprises of two nearly median chromosomes (3 and 4), one nearly submedian (-) chromosome (1) and one nearly subterminal (-) chromosome (2).

Group two consists of the medium sized chromosomes numbered 5 to 14 and $\mathrm{X}$ chromosome. It comprises of four nearly median chromosomes $(6,7,13$, and X), one nearly submedian $(+)$ chromosome (9), and six nearly submedian (-) chromosomes $(5,8,10,11,12$ and 14$)$.

Group three consists of the small sized chromosomes numbered 15 to 18 and $\mathrm{Y}$ chromosome. It comprises of one median chromosome (15), one nearly median chromosome (Y), two nearly submedian (-) chromosomes (16 and 17), and one terminal chromosome (18).

Similarly, ideograms were accurately constructed using the measurements of the chromosomes as shown in figures 3 and 4 . In figure 3 which shows the size variation of the chromosomes, it is seen that there is a somewhat sharp break in chromosome lengths between chromosomes 4 and 5, and thereafter, there is a gradual decrease in size. In figure 4 which shows the variation of the centromeric location, it appears that there is greater variation in the lengths of the long (q) arms than in the short (p) arms.
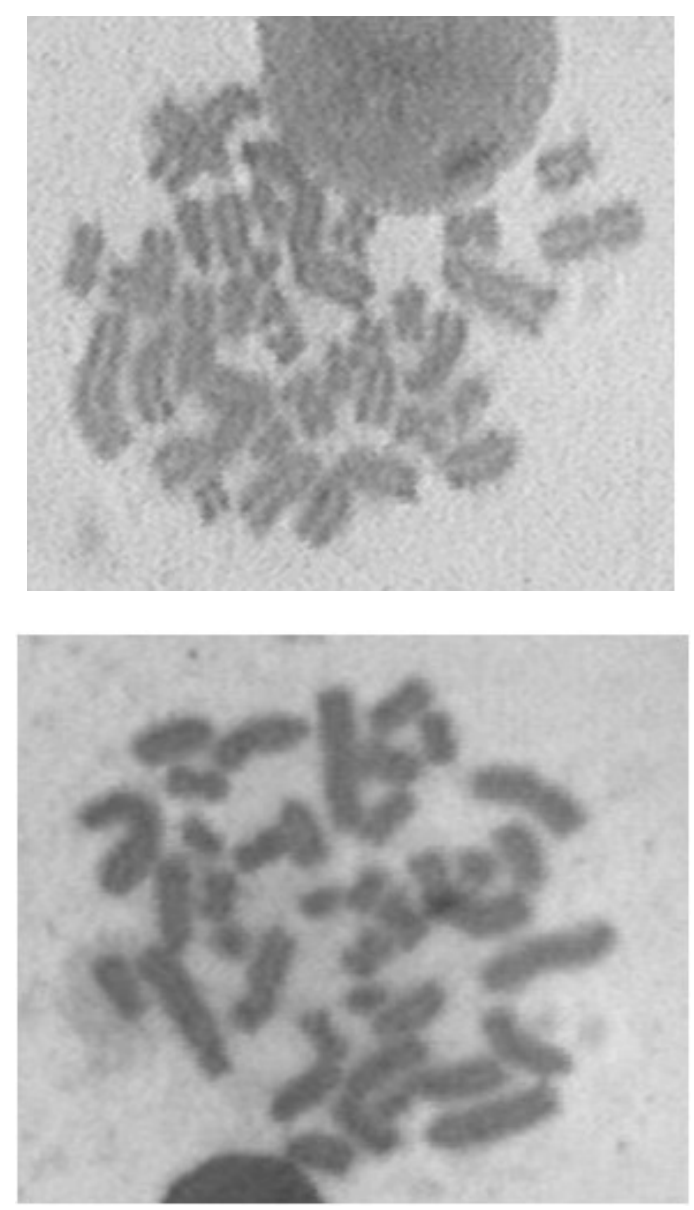

Fig. 1: Mitotic metaphase chromosomes of a male Rattus rattus. 


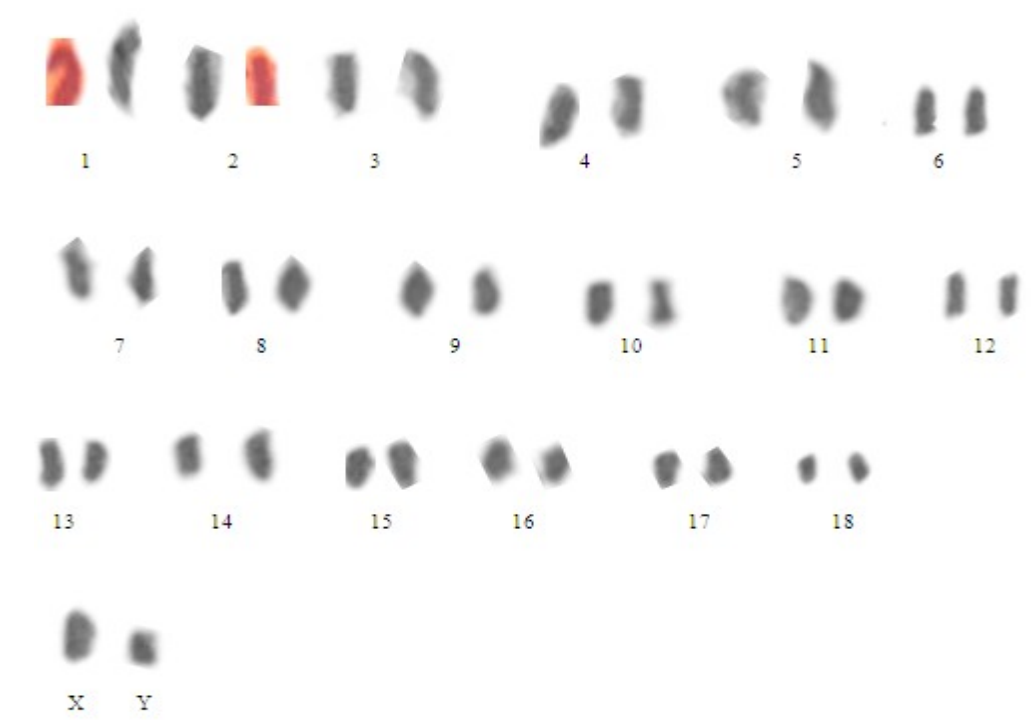

Fig. 2: The karyotype (idiogram) of the male Rattus rattus prepared from the same spread shown in fig. 1.

Table 2: The Nomenclature of the chromosomes of Rattus rattus, using the Centromeric indices. The Centromeric Index (i) for each chromosome was derived from the formular, $\mathrm{i}=100 \mathrm{~S} / \mathrm{C}$ where $\mathrm{S}$ and $\mathrm{C}$ represent the short arm length and total length respectively. The chromosome lengths were measured in microns $(\mu)$

\begin{tabular}{|c|c|c|c|c|c|}
\hline Chromosome number & $\begin{array}{l}\text { Short arm } \\
\text { length (S) }\end{array}$ & $\begin{array}{l}\text { Long arm } \\
\text { length (L) }\end{array}$ & Total length (C) & $\begin{array}{l}\text { Centromeric index } \\
\text { (i) }\end{array}$ & Nomenclarure \\
\hline 1 & 12.0 & 31.5 & 43.5 & 27.58 & Nearly submedian(-) \\
\hline 2 & 6.9 & 34.4 & 41.3 & 16.70 & Nearly subterminal(-) \\
\hline 3 & 14.3 & 22.4 & 37.1 & 39.62 & Nearly median \\
\hline 4 & 15.1 & 17.1 & 32.2 & 46.89 & Nearly median \\
\hline 5 & 8.1 & 19.1 & 27.2 & 29.77 & Nearly submedian(-) \\
\hline 6 & 10.4 & 15.5 & 25.9 & 40.1 & Nearly median \\
\hline 7 & 8.5 & 15.6 & 24.1 & 38.2 & Nearly median \\
\hline 8 & 8.3 & 13.9 & 22.2 & 37.38 & Nearly submedian(-) \\
\hline 9 & 4.4 & 17.2 & 21.6 & 20.37 & Nearly submedian $(+)$ \\
\hline 10 & 6.9 & 13.9 & 20.8 & 33.17 & Nearly submedian(-) \\
\hline 11 & 6.2 & 12.6 & 18.8 & 32.97 & Nearly submedian(-) \\
\hline 12 & 6.4 & 10.8 & 17.2 & 37.20 & Nearly submedian(-) \\
\hline 13 & 7.2 & 9.2 & 16.4 & 43.90 & Nearly median \\
\hline 14 & 5.0 & 10.8 & 15.8 & 31.64 & Nearly submedian(-) \\
\hline 15 & 6.95 & 6.95 & 13.9 & 50 & Median \\
\hline 16 & 3.2 & 9.2 & 12.4 & 25.80 & Nearly submedian(-) \\
\hline 17 & 2.5 & 8.9 & 11.4 & 21.92 & Nearly submedian(-) \\
\hline 18 & 0.00 & 9.9 & 9.9 & 0.00 & Terminal \\
\hline $\mathrm{X}$ & 9.8 & 11.4 & 21.2 & 46.22 & Nearly median \\
\hline $\mathrm{Y}$ & 5.6 & 6.4 & 12.0 & 46.66 & Nearly median \\
\hline
\end{tabular}

$(-)$ indic-*ates that centromere is away from terminal position

$(+)$ indicates that centromere is nearer to terminal position 


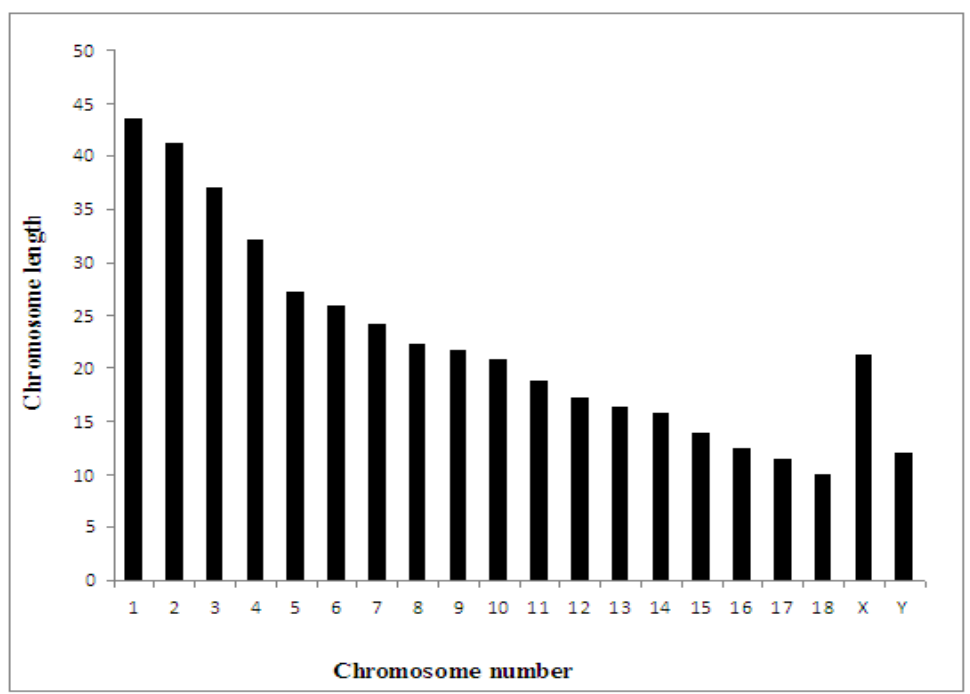

Fig. 3: An ideogram of the karyotype of Rattus rattus showing the size variation among the chromosomes. The relative chromosome lengths were obtained from the mean of 5 well spread and representative metaphase cells.

Each relative chromosome length is a percentage of the sum total of the lengths of the whole chromosome complement.

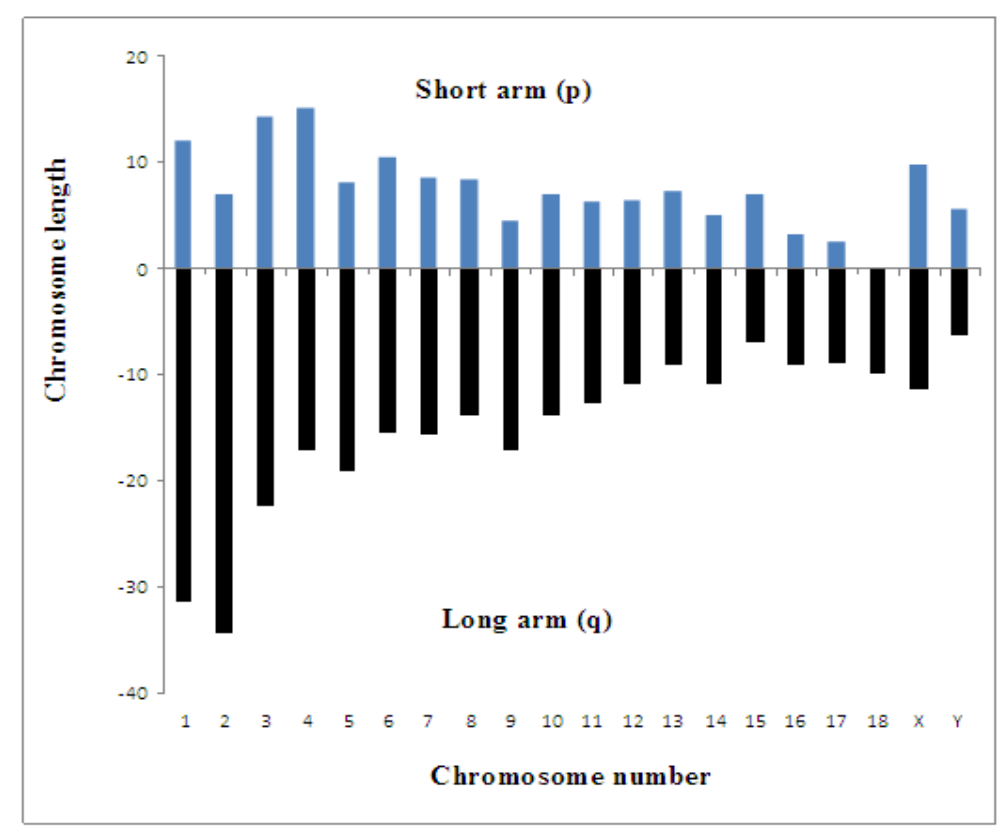

Fig. 4: An idiogram of the karyotype of Rattus rattus showing the morphology of the chromosomes. The idiogram was constructed from the same measurements used for figure 3 . The 0 represents the position of the centromere.

\section{Discussion And Conclusion}

The local Black Rat, Rattus rattus, karyotyped in this study has a diploid chromosome number of $2 n=$ 38. This agrees with the findings made on this same race in South America [10] and in Italy [11], [12]. However, the reporting of a diploid number of $2 \mathrm{n}=42$ for the Rattus rattus race in Japan [4], [5], [6], [7], in Switzerland [8] and in Malaysia [9] is very curious. It thus appears as a case of misrepresentation. This is more so as the observation of the diploid number of $2 n=38$ as observed in the present study, coincides with the advent of better extraction methods. Nevertheless, the present scenario poses a great challenge to cytotaxonomy. This is because it raises a doubt about the true cytotaxonomic status of these earlier black rat populations karyotyped. The need, therefore, arises for a re-visitation of the karyology of these black rat populations in Japan, Switzerland and Malaysia. This will go a long way in establishing the true taxonomic status of the Black rat populations the world over. 


\section{References}

[1]. Wikipedia (2009). Rats. Wikipedia Foundation Incorporated.15pp. $>$ http://www.wikipedia. com< $<$ Accessed December 23, 2009.

[2]. Jung, Y. Park, G. M. and Chung, P. R., Chromosome study of two similar lymnaeid snail species, Korean Austropeples ollula and an exotic species in Australia (Pulmonata: Lymnaeidae), Korean Journalof Malacology, 18 (1), 2002,61 - 65.

[3]. Dorothy, A. M., Vaithilingam, G. D., Carmia, B. and Orlando, J. M., The quinacrine fluorescent and Giemsa banding karyotype of the Brown Rat, Rattus norvegicus, and banded chromosome analysis of transformed and malignant rat liver cell lines, Cancer Research, 32, 1972, $2375-2382$.

[4]. Oguma, K., Karyological studies of the Black Rat, Rattus rattus. Journal of the Faculty of Science, Hokkaido University, 4, $1935,35-39$.

[5]. Tateishi, S., Chromosomal studies of the species of the Black Rat, Rattus rattus,. Proceedings of the Japanese Association for the Advancement of Science, 10, 1935, 1007 - 1112.

[6]. Makino, S., The karyotype of the Japanese population of the Black Rat, Rattus rattus, Journal of the FacultyofScience, Hokkaido University, 9, 1953, $19-23$.

[7]. Yoshida, T. H., Nakamura, A. and Fukaya, T., The chromosome number of the Black Rat, Rattus rattus, Chromosoma, 16, 1965, 70 -73 .

[8]. Matthey, R., Chromosome studies of Swiss population of the Black Rat, Rattus rattus, Experientia, 12, $1956,337-341$.

[9]. Yong, H. S., The karyotype of the Malaysian population of the Black Rat, Rattusrattus. Chromosoma, $16,1969,70-74$.

[10]. Bianchi, O. N., Paulette-Vanrell, J. and De Vidal Rioja, L. A., The karyotype of the Black Rat, Rattus rattus, of South American population, Experientia, 25, 1969, $1111-1115$.

[11]. Capanna, E. and Civitelli, M. V., Review of mammalian chromosomes, Chromosomal Newsletter, 10, $1969,85-91$.

[12]. Capanna, E., Civitelli, M. V. and Nezer, R., The karyotype of the Black Rat, Rattus rattus, Cellular and Molecular Life Sciences, 26 (4), 1970, $422-425$.

[13]. Adegoke, J. A. and Ejere, V. C., Despcription of the chromosomes of three lizard species belonging to the genus Mabuya (Scincidae, Reptilia). Caryologia, 44 (3), 1991, $333-342$.

[14]. Ejere, V. C. and Adegoke, J. A., Aspects of the reproductive ecology of tropical lizards: Seasonal behaviour of meiotic chromosomes in Agama agama agama L, (Agamidae, Reptilia), The Zoologist, 1 (1), 2002, 86 - 94.

[15]. Abraham, Z. and Prasad, P. N., A system of chromosome classification andnomenclature, Cytologia, 48, $1983,95-101$. 